\title{
Performance and Comparative Analysis of Wired and Wireless Communication Systems using Local Area Network Based on IEEE 802.3 And IEEE 802.11
}

\author{
*1OKPEKI, UK; ${ }^{2}$ EGWAILE, JO; ${ }^{2}$ EDEKO, F
}

${ }^{*}$ Department of Electrical and Electronic Engineering, Delta State University, Abraka, Oleh Campus, Delta State, Nigeria.

${ }^{2,}$ Department of Electrical and Electronic Engineering, University of Benin, Benin - City, Edo State, Nigeria. *Corresponding AuthorEmail: ufuomakazeem@gmail.com,ukokpeki@delsu.edu.ng

\begin{abstract}
The aim of this research is to carryout Performance Analysis and Comparison of Wired and Wireless Communication Systems using Local Area Network (LAN) based on IEEE 802.3 and IEEE 802.11 standard, carried out with emphasis on Throughput, Delay, Bit error rate and Signal to Noise Ratio by collecting data at the Delta State University e - library network.. From the experimental results of the ten shots sample data for both wired and wireless networks, the wired network in its three transmission protocols (TCP, IPV4 \& IPV6) has overall throughput average of $6085 \mathrm{Kbps}$, while the wireless has overall throughput average of $52752 \mathrm{Kbps}$. From the computed total average values, the wired network exhibited delays of $4 \mathrm{~ms}, 45 \mathrm{~ms}$ and $6 \mathrm{~ms}$ in its (TCP, IPV4 \& $6 \mathrm{~ms}$ ) respectively with overall average of 52 milliseconds (52ms). While on the other hand the wireless had delays of 36ms, $4 \mathrm{~ms} \& 52 \mathrm{~ms}$ in its (TCP, IPV4 \& IPV6) respectively, with overall average of 57 milliseconds $(57 \mathrm{~ms})$. In terms of Bit Error Rate, the wired network have bit error rate of $1.364 \mathrm{E}-03 \%, 7.773 \mathrm{E}-05 \%$ and $7.28 \mathrm{E}-06 \%$ in its (TCP, IPV4 and IPV6) respectively, with overall average of $4.83003 \mathrm{E}-04 \%$. While the wireless network have the values of $8 .-7 \mathrm{E}-05 \%, 0 \%$ and $7.61 \mathrm{E}-04 \%$ in its (TCP, IPV4 and IPV6) respectively, with overall average of $2.805667 \mathrm{E}-04 \%$. With respect to Signal to Noise Ratio, the wired network have signal to noise ratio overall average of $8.266 \mathrm{~dB}$. While the wireless network have the overall average of $5.178 \mathrm{~dB}$. Based on the, networks performance metrics statistical data analyzed above for both wired and wireless, we are of the opinion that the wireless network is preferable to the wired network under the area investigated.
\end{abstract}

\section{DOI: https://dx.doi.org/10.4314/jasem.v22i11.3}

Copyright: Copyright (C 2018 Okpeki et al. This is an open access article distributed under the Creative Commons Attribution License (CCL), which permits unrestricted use, distribution, and reproduction in any medium, provided the original work is properly cited.

Dates: Received: 02 October 2018; Revised: 26 November 2018; Accepted 30 November 2018

Keywords: Performance, Communication systems, IEEE 802.11 and IEEE 802.3 Standards.

Communication in its simplest form is the transmission of information from one point to another via a medium either wired or wireless and is a bidirectional process (Anokh and Chhabra, 2007). Wired networks establish connection between various devices through connecting media such as cables and routers. Whether wired or wireless communication systems, all have network topology, which is the schematic form of switching elements, transmission links, routers and other peripherals. Network topologies are categorized into two distinct classes namely: physical network layout and logical network layout (Rajput, 2009). Wireless local area networks are commonly implemented using the Institute of Electrical and Electronic Engineers (IEEE) 802.11 standard. Wider area coverage is made possible by utilizing General Packet Radio Service (GPRS) the existing mobile phone infrastructure for the transmission. The integration between packet use and voice communication further evolved with the deployment of Third Generation and Universal Mobile Telecommunications Service (3G/UMTS), (Keren, 2011). This system is specially designed to carry packet data, video and voice communication with much higher capability, than previous wide area coverage networks. With the adoption of network technologies for the purpose of, education, business, banking and defense etc, these interconnected set of computer systems permits interactive resource sharing between connected pair of systems (Sharma, 2007). Rapid advances have taken place in the field of wired and wireless networks. The traditional wired transmission medium provides high speed connectivity but poses constraints like immobility and extensive cabling. Wireless communication is a flexible data communication system implemented as an extension to or as an alternative to wired communication (Randhawa and Hardy, 2002). The wireless technologies employ infrared, spread spectrum and microwave radio transmission techniques with varying data rates. Though wireless technology provides convenience and advantages like ease of mobility, scalability and flexibility, it has certain drawbacks like speed, range, reliability, security, bit error rate (BER) and hidden terminal problems (Tamar, 2000). The Wireless Local Area 
Network $\left(\mathrm{W}_{\mathrm{L}} \mathrm{LAN}\right)$ is based on IEEE 802.11 standard using Carrier Sense Multiple Access with Collision Avoidance (CSMA/CA) MAC protocol as access method. The wired Local Area Network (W based on IEEE 802.3 (Ethernet) standard with carrier sense medium access with collision detection (CSMA/CD) MAC protocol, as access method.. In this research work the performance analysis of wired and wireless communication networks was carried out using some performance metrics, such as throughput, Packet Delay, Bit Error Rate (BER) and Signal to Noise Ratio (SNR) as a basis for comparison. In more recent times most researches on network performance were centred on TCP. A holistic view encompassing both throughput, delay and bit error rate in TCP, IPV4 and IPV6 remained relatively unstudied and this motivated this work. Several works have been done in different aspects of performance characterization of the IEEE 802 Standards. High quality services in both wired and wireless networks environment, accurate tracking and location prediction is one of the ways to significantly improve the performance and reliability of networks protocols and infrastructure. Satish et al, (2012) compared wired and wireless networks in the area of installation, cost reliability and performance.

The performance parameters evaluated were throughput, data dropped, traffic received and collision counts. The authors analyzed the wired and the wireless networks using OPNET simulator but, no physical measurement were made. In Rahul et al (2011), the author centered his performance analysis of wired and wireless computer networks on congestion control mechanism. The congestion control mechanism, is an important issue in designing any good network. The congestion control involves two factors that measures the performance of networks, i.e. delay and Throughput and the author analyzed the two performance metrics using OPNET simulator .This work was purely simulation. Traffic patterns have significant impact on network performance. Analytical models for the performance evaluation of wired interconnection networks and integrated wireless network have been widely reported. However, most of these models are developed under simplified assumption of non-bursty poison process with uniform distributed message destinations. In light of the above, the author developed analytical model and propose it to evaluate end to end delay and throughput of wired and wireless local area networks under traffic patterns exhibited by real word applications. (Yulei, 2010; Dhobale et al, 2014), investigated the performance of wired and wireless networks. The performance of both networks were evaluated on the basis of a common parameter, throughput, to know how both networks behaves. The evaluation analysis were done using
OPNET simulation environment. In (Rahul et al, 2010), the authors carried out performance analysis of IEEE802.11b wireless and IEEE802.3 wired LANs standards using soft computing techniques for their performance comparison by varying the attributes of network objects such as traffic loads, file size, RTS/CTS, customizing the physical characteristics to vary BER, slot time to determine their impact on throughput and delay.. In (Salam et al, 2007), the authors evaluated the performance of IEEE 802.116 wireless LAN applied in E-learning classroom. They used OPNET IT 9.1 simulator in their simulations to study E-learning classroom area network scenario. They build a model of browsing behavior of E-learning and web client and investigated the performance of Elearning classroom area network based on these performance metrics, delay, throughput and web object size. And their results showed that IEEE 802.11b WLAN have a minimum delay, high throughput and can support up to 50 clients. In (Abdul etal, 2006), the authors carried out performance comparison of TCP and UDP over wired and wireless LAN. They used DSDV routing protocols to evaluate their performance. They compared TCP and UDP in terms of throughput using network simulator-2. Their results showed that the wired network has better performance than the wireless network in terms of throughput. The objective of this paper is to evaluate the performance of wired and wireless communication systems by carrying out measurement of throughput, delay and bit error rate in both networks.

\section{MATERIALS AND METHOD}

The measurement methodology was employed in the research and the Delta State University e-library wired and wireless networks infrastructure were used to carried out the measurements.

The Wired and the wireless networks has (10) computers (HP Compaq 4000 pro-small form factor, HP LE 1901w) each. The wired network consists of three CISCO switches SF-100-24, 24-port 10/100 switch, two serves, Mikrotip server, a window server, one CISCO 1800 Series Router, while the wireless network consists of three cam baseline switches 2024 model 3C16471, a server (Evolution Idirect), one wireless router (CISCO 1800 series). Three measurements software were used namely: Wireshark, Matlab and Microsoft Excel. The three software were installed in Advent laptop computer (window vista), that is compatible with windows operating systems. Wireshark 1.6.7 version was used to conduct the measurements, Matlab version R2016a was used for the modeling. Microsoft Excel was used for the performance metrics computations. 
Data Collections and Computations: Measurements were taken in the morning, afternoon and evening on a continuous basis as could be accommodated. However since the data are not time series data, the time of taking the measurements is of negligible importance. Thereafter, the ten workstations designated for the data collection started surfing or browsing the internet for two hours every day for a period of six months. Ten sample data were captured for both network in their TCP, IPV4 and IPV6 transmission protocols being investigated

\section{RESULTS AND DISCUSSIONS}

The results of network modeling of Throughput (T), Delay (D) and Bit Error Rate (BER) are presented in table 1, 2 and 3. From the tables the results validated the primary data computed for Throughputs, Delay and Bit Error Rate for (TCP, IPV4 and IPV6) for the ten shots of both wired and wireless networks. The average values of throughputs in (TCP, IPV4 \& IPV6) for both wired and wireless networks are presented in Table 4, 5. From table 4 and 5 above, the wired network in its three transmission protocols, (TCP, IPV4 \& IPV6) has Throughputs values of $15^{*} 10^{3} \mathrm{Kbps}$, $21 * 10^{2} \mathrm{Kbps}$ and $85^{*} 10^{1} \mathrm{Kbps}$ respectively, with overall average of $60 * 10^{2} \mathrm{Kbps}$, while the wireless has Throughputs values of $15^{*} 10^{4} \mathrm{Kbps}, 39 * 10^{2} \mathrm{Kbps}$ and $54 * 10^{1} \mathrm{Kbps}$ in its, (TCP, IPV4 \& IPV6) respectively, with an overall average of $52 * 10^{3} \mathrm{Kbps}$.

Table 1: Average Values of Throughput for the Ten Shots (Wired and Wireless Network)

\begin{tabular}{lllllll}
\hline & \multicolumn{2}{c}{ Throughput Wired } & \multicolumn{2}{c}{ Throughput Wireless } \\
\hline & TCP & IPV 4 & IPV 6 & TCP & IPV 4 & IPV 6 \\
\hline Shot 1 & $28 * 10^{2}$ & $33 * 10^{2}$ & $44 * 10^{2}$ & $28 * 10^{3}$ & $19 * 10^{2}$ & $73 * 10^{1}$ \\
Shot 2 & $44 * 10^{3}$ & $98 * 10^{2}$ & $25 * 10^{1}$ & $25 * 10^{3}$ & $15 * 10^{2}$ & $12 * 10^{2}$ \\
Shot 3 & $14 * 10^{2}$ & $76 * 10^{1}$ & $55 * 10^{1}$ & $34 * 10^{3}$ & $33 * 10^{2}$ & 65 \\
Shot 4 & $12 * 10^{2}$ & $80 * 10^{1}$ & $35 * 10^{1}$ & $33 * 10^{3}$ & $27 * 10^{2}$ & 59 \\
Shot 5 & $95 * 10^{2}$ & $43 * 10^{2}$ & $35 * 10^{1}$ & $77 * 10^{3}$ & $28 * 10^{2}$ & $15 * 10^{1}$ \\
Shot 6 & $84 * 10^{2}$ & $12 * 10^{2}$ & 97 & $13 * 10^{3}$ & $18 * 10^{2}$ & 53 \\
Shot 7 & $79 * 10^{3}$ & $62 * 10^{1}$ & $23 * 10^{1}$ & $19 * 10^{3}$ & $11 * 10^{3}$ & 64 \\
Shot 8 & $13 * 10^{2}$ & $40 * 10^{1}$ & 41 & $12 * 10^{5}$ & $64 * 10^{2}$ & $15 * 10^{2}$ \\
Shot 9 & $21 * 10^{1}$ & $22 * 10^{1}$ & $15 * 10^{2}$ & $21 * 10^{3}$ & $26 * 10^{2}$ & $11 * 10^{2}$ \\
Shot 10 & $30 * 10^{2}$ & $29 * 10^{1}$ & $73 * 10^{1}$ & $10 * 10^{3}$ & $48 * 10^{2}$ & $32 * 10^{1}$ \\
\hline
\end{tabular}

Table 2: Average Values of Delay for the Ten Shots in (Wired and Wireless Network)

\begin{tabular}{lllllll}
\hline & Delay Wired & \multicolumn{3}{c}{ Delay Wireless } \\
\hline & TCP & IPV 4 & IPV 6 & TCP & IPV 4 & IPV 6 \\
\hline Shot 1 & 3 & 10 & 13 & 2 & 8 & 13 \\
Shot 2 & 2 & 15 & 18 & 2 & 5 & 97 \\
Shot 3 & 3 & 42 & 41 & 1 & 5 & 49 \\
Shot 4 & 5 & 80 & 63 & 1 & 4 & 18 \\
Shot 5 & 4 & 58 & 78 & 1 & 5 & 31 \\
Shot 6 & 4 & 20 & 44 & 1 & 5 & 34 \\
Shot 7 & 9 & 31 & 33 & 3 & 6 & 146 \\
Shot 8 & 5 & 55 & 52 & 29 & 0 & 59 \\
Shot 9 & 3 & 26 & 38 & 1 & 3 & 57 \\
Shot 10 & 4 & 28 & 71 & 1 & 4 & 21 \\
\hline
\end{tabular}

Table 3: Average Values of Bit Error Rate for the Ten Shots in (Wired and Wireless Network)

\begin{tabular}{lllllll}
\hline & \multicolumn{3}{c}{ Bit Error Rate Wired } & \multicolumn{3}{c}{ Bit Error Rate Wireless } \\
\cline { 2 - 7 } & TCP & IPV 4 & IPV 6 & TCP & IPV 4 & IPV 6 \\
\hline Shot 1 & $14 * 10^{-3}$ & $15 * 10^{-4}$ & $38 * 10^{-4}$ & 0 & $17 * 10^{-6}$ & $71 * 10^{-4}$ \\
Shot 2 & $23 * 10^{-3}$ & $31 * 10^{-6}$ & $34 * 10^{-6}$ & 0 & $10 * 10^{-6}$ & $15 * 10^{-3}$ \\
Shot 3 & $88 * 10^{-4}$ & $66 * 10^{-6}$ & $13 * 10^{-6}$ & 0 & $61 * 10^{-7}$ & $61 * 10^{-4}$ \\
Shot 4 & $19 * 10^{-3}$ & $24 * 10^{-4}$ & $26 * 10^{-6}$ & 0 & $25 * 10^{-7}$ & $59 * 10^{-4}$ \\
Shot 5 & 0 & $11 * 10^{-4}$ & $95 * 10^{-6}$ & 0 & $44 * 10^{-7}$ & $56 * 10^{-4}$ \\
Shot 6 & $94 * 10^{-4}$ & $68 * 10^{-6}$ & $19 * 10^{-6}$ & 0 & $89 * 10^{-7}$ & $57 * 10^{-4}$ \\
Shot 7 & $23 * 10^{-3}$ & $22 * 10^{-6}$ & $32 * 10^{-6}$ & 0 & $1 * 10^{-6}$ & $60 * 10^{-4}$ \\
Shot 8 & $57 * 10^{-7}$ & $32 * 10^{-6}$ & $23 * 10^{-6}$ & 0 & $40 * 10^{-7}$ & $59 * 10^{-4}$ \\
Shot 9 & $16 * 10^{-3}$ & $17 * 10^{-6}$ & $18 * 10^{-6}$ & 0 & $28 * 10^{-7}$ & $61 * 10^{-4}$ \\
Shot 10 & $20 * 10^{-3}$ & $60 * 10^{-6}$ & $11 * 10^{-4}$ & 0 & $54 * 10^{-7}$ & $11 * 10^{-3}$ \\
\hline Comparison of Wired and Wireless Networks Throughput Efficiency in $($ TCP, IPV4 and IPV6
\end{tabular}

Table 4: Average Value of Throughput for Wired and Wireless Networks in their Three Transmission Protocols

\begin{tabular}{llllll}
\hline Wired & \multicolumn{5}{c}{ Wireless } \\
\hline TCP & IPV4 & IPV6 & TCP & IPV4 & IPV6 \\
$15 * 10^{3}$ & $21 * 10^{2}$ & $85 * 10^{1}$ & $15 * 10^{4}$ & $39 * 10^{2}$ & $54 * 10^{1}$ \\
\hline \multicolumn{4}{l}{ Average $=60 * 10^{2} \mathrm{Kbps}$} & \multicolumn{4}{l}{ Average $=52 * 10^{3} \mathrm{Kbps}$} \\
\hline
\end{tabular}


Table 5: Average Throughput Efficiency

\begin{tabular}{llllll}
\hline \multicolumn{5}{c}{ Wired } & \multicolumn{5}{c}{ Wireless } \\
\hline TCP & IPV4 & IPV6 & TCP & IPV4 & IPV6 \\
\hline $23 \%$ & $32 \%$ & $62 \%$ & $77 \%$ & $68 \%$ & $38 \%$ \\
\hline
\end{tabular}

From the average throughput efficiency table, the wired network has $23 \%, 32 \%$ \& $62 \%$ respectively in its (TCP, IPV4 \& IPV6) transmission protocols, with mean statistics of $39 \%$, while the wireless has $77 \%$, $68 \%$ \& $38 \%$ respectively in its (TCP, IPV4 \& IPV6) transmission protocols, with mean statistics of $61 \%$. The up and down in the network throughput are due to signal fluctuation and load variability, intermittent connectivity and packet loss due to channel interference.

Comparison of Delay in Wired and Wireless Networks: The average delays in the three transmission protocols (TCP, IPV4 \& IPV6) of both wired and wireless networks are presented in table 6 .

Table 6: Average Delay of Wired and Wireless Networks in their Three Transmission Protocols

\begin{tabular}{llllll}
\hline Wired & \multicolumn{4}{c}{ Wireless } \\
\hline TCP & IPV4 & IPV6 & TCP & IPV4 & IPV6 \\
\hline 4 & 45 & 6 & 36 & 4 & 52 \\
\hline \multicolumn{3}{l}{ Average $=52 \mathrm{MS}$} & \multicolumn{3}{c}{ Average $=57 \mathrm{MS}$} \\
\hline
\end{tabular}

Table 7: Average Bit Error Rate for Wired and Wireless Networks in their Three Transmission Protocols

\begin{tabular}{lcllll}
\hline Wired & \multicolumn{5}{l}{ Wireless } \\
\hline TCP & IPV4 & IPV6 & TCP & IPV4 & IPV6 \\
\hline $13 * 10^{-4}$ & $77 * 10^{-6}$ & $72 * 10^{-7}$ & $8 * 10^{-7}$ & 0 & $76 * 10^{-5}$ \\
\hline Average $=48 * 10^{-5} \%$ & & Average $=28 * 10^{-5} \%$ & \\
\hline
\end{tabular}

Table 8: Signal-to-Noise Ratio (SNR) Networks Results

\begin{tabular}{llllll}
\multicolumn{5}{c}{ Table 8: Signal-to-Noise Ratio (SNR) Networks Results } \\
\hline Wired & \multicolumn{5}{l}{ Wireless } \\
\hline TCP & IPV4 & IPV6 & TCP & IPV4 & IPV6 \\
\hline $65 * 10^{-3} \mathrm{~dB}$ & $85 * 10^{-3} \mathrm{~dB}$ & $97 * 10^{-3} \mathrm{~dB}$ & $85 * 10^{-3} \mathrm{~dB}$ & 0 & $70 * 10^{-3} \mathrm{~dB}$ \\
\hline Average $=82 * 10^{-2} \mathrm{~dB}$ & & Average $=51 * 10^{-2} \mathrm{~dB}$ & \\
\hline
\end{tabular}

In table 6 above, the wired network exhibited delays of $4 \mathrm{~ms}, 45 \mathrm{~ms}$ and $6 \mathrm{~ms}$ in its (TCP, IPV4 \& 6ms) respectively with overall average of 52 milliseconds $(52 \mathrm{~ms})$. While on the other hand the wireless had delays of $36 \mathrm{~ms}, 4 \mathrm{~ms} \& 52 \mathrm{~ms}$ in its (TCP, IPV4 \& IPV6) respectively, with overall average of 57 milliseconds (57ms).

Comparison of Bit Error Rate for Wired and Wireless Networks: The average Bit Error Rates for wired and wireless networks in their (TCP, IPV4 and IPV6) are presented in table 7 .

From the table 7 above the wired network have bit error rate of $13 * 10^{-4} \%, 77 * 10^{-6} \%$ and $72 * 10^{-7} \%$ in its (TCP, IPV4 and IPV6) respectively, with overall average of $48 * 10^{-5} \%$. While the wireless network have the values of $8 * 10^{-6} \%, 0 \%$ and $76 * 10^{-5} \%$ in its (TCP, IPV4 and IPV6) respectively, with overall average of $28 * 10^{5} \%$. These errors were due to intermittent connectivity and packet drops due to channel interference.
Comparison of Signal to Noise Ratio for Wired and Wireless Networks: The average signal to noise ratio computed for wire and wireless networks are presented in table 8.From table 8 above the wired network have signal to noise ratio of $65 * 10^{-3} \mathrm{~dB}, 85$ $* 10^{-3} \mathrm{~dB}$ and $97 * 10^{-3} \mathrm{~dB}$ in its (TCP, IPV4 and IPV6) respectively, with overall average of $82 * 10^{-2}$ $\mathrm{dB}$. While the wireless network have the values of 85 $* 10^{-3} \mathrm{~dB}, 0 \mathrm{~dB}$ and $70 * 10^{-2} \mathrm{~dB}$ in its (TCP, IPV4 and IPV6) respectively, with overall average of $51 * 10^{-}$ ${ }^{2} \mathrm{~dB}$. The variation in the signal strength was due to signal fluctuation and load variability in the network

Conclusion: From the performance analysis and comparison of wired and wireless communication systems networks some of the findings, will help establishments, institutions and organizations in making choice of network deployment. Also it will help network engineers in making choice of throughput predicting model in predicting throughputs of the infrastructure and it will enhance network service providers in implementing state of the art equipment that will boost their performances. 
Acknowledgement: The authors wish to acknowledge the kind support of the Education Trust Fund (ETF) through a grant provided to Okpeki ufuoma kazeem

\section{REFERENCES.}

Abdul, RR; Khurram, S; Abdul, QM (2006), Evaluation and Comparison of TCP and UDP over Wired-Cum-Wireless LAN. Research Gate: 337-342 https://researchgate.net/publication/259335727

Akintola, AA; Aderounmu, GA; Owojori, AA; Adigun, AA (2006), Performance Modeling of UDP Over IP-Based Wired and Wireless Networks. Issues in Info. Techn. V(3): $13-21$

Dhobale, JV; Kalyankar, NV; Khamitkar, SD (2014), Computer Network Performance Evaluation Based on Different Data Packet Size Using OMNeT++ Simulation Environment Int. J. Adv. Res. Compt. Sci Techn. V(2.)2: $91-95$

Keren, T (2011), Large Scale Wireless Local Area Network Measurement and Privacy Analysis." Dartmouth College. Hannover. New Hampshire. $\mathrm{P}_{\mathrm{P}} 23$

Rahul, M; Vikas, G; Bansal, RK (2011), Simulation and Performance Analysis of Wired and Wireless Computer Networks. Glob. J. Comp. Sci. Technol. V(11)10: $11-17$

Rahul Malhotra and Bansal R. K (2010). Performance Analysis of Wired and Wireless LAN Using Soft Computing Techniques. Glob. J. Compt. Sci. Techn. V(10)7: 67 -71
Randhawa, TS; Hardy, S (2002), Network Management in Wired and Wireless Network. (book review): IEEE Comm. Mag. V(40)5: 30-32

Rajput, RK (2009), Electronics and Communications Engineering, Birla publications PVT Ltd. $\mathrm{P}_{\mathrm{P}} 200$

Salam, AN; Ibrahim, MME; Samir, MS (2007) Performance Evaluation of Wireless IEEE 802.11b used for E-learning Classroom Network. IAENG Int. J. Compt. Sci. 34:1, IJCS_34_1_7.

Satish, KS; Sonal, JR; Dharmistha, DV (2012), Performance Evaluation of Wired and Wireless Local Area Networks. Int. J. Engn. Res. Dev. V(1)11: 43-48.

Sharma, S (2007), Wireless and Cellular Communications. $\mathrm{P}_{\mathrm{P}} 150$

Tamer, MSK (2000), Performance Analysis of Wireless Local Area Network $P_{P} 45$

Tanja, L; Daniel, F; Arek, D (2003), “TCP throughput over links with high bit error Rate," C.McDonald (ed.) Converge Networking. Int. Fed. Info. Proc. Retrieved 10/20/2016.

Yulei, W (2010), Performance Modelling and Evaluation of Heterogeneous Wired/Wireless Network under Bursty Traffic. Thesis for the degree of Doctor of philosophy, School of Computing, Informatics and Media, University of Bradford. $\mathrm{P}_{\mathrm{P}} 66$ 